\title{
Use of Negative Bias Potential for High Throughput Array Tomography in an Integrated Light-Electron Microscope
}

\author{
Ryan Lane $^{1 *}$, Yoram Vos ${ }^{1}$, Pascal de Boer ${ }^{2}$, Ben N.G. Giepmans ${ }^{2}$ and Jacob P. Hoogenboom ${ }^{1}$ \\ ${ }^{1}$ Imaging Physics, Delft University of Technology, Delft, The Netherlands. \\ ${ }^{2}$ Cell Biology, University Medical Centre Groningen, Groningen, The Netherlands. \\ * Corresponding author: r.i.lane@tudelft.nl
}

Volume electron microscopy (EM) has progressively become a driving force in exploring and analysing three-dimensional biological structures across ever-increasing spatial scales. While advances in technology and automation have revolutionized imaging capabilities, low throughput persists as the primary limitation to achieving larger and larger volumes [1]. One strategy for increasing throughput is to combine a fluorescence and electron microscope together into one integrated system. Doing so provides the advantage of being able to use fluorescence expression as a guide for selecting regions of interest for high resolution EM imaging [2].

One drawback of an integrated approach, however, is the inability to apply a post-staining agent for higher contrast. Typically in EM, and especially for (S)TEM imaging, biological samples are stained with relatively high concentrations of heavy metals such as uranyl, osmium, and lead in order to effectively scatter the high energy electrons. In conventional EM imaging this gives a higher backscatter yield, the results of which are high-SNR images exhibiting excellent contrast [3]. Unfortunately, applying such heavy metal stains to immunolabelled samples has the unfavourable consequence of quenching fluorescence. Samples intended for use in the integrated microscope are therefore stained with lesser concentrations, resulting in relatively weaker contrast. Ordinarily this effective loss of signal would be compensated for by scanning with longer electron beam exposures-and therefore longer overall acquisitions. Of course since one of the primary advantages of using an integrated microscope is to maximize throughput, settling for higher acquisition times is highly undesirable.

An alternate means for compensating weaker signal is to increase the BSE yield by accelerating the backscatter electrons to the detector. This can be done by e.g. biasing the sample with a negative potential. While this causes an acceleration for the backscatter electrons, the primary electrons experience a retarding electric field in their trajectory towards the sample. By adjusting the primary beam energy together with the negative potential applied to the sample, the landing energy can be tuned to achieve the appropriate interaction volume, effectively optimizing the BSE signal [4].

Applying a negative bias to the sample in an integrated microscope does, however, present certain challenges. In particular, the high-NA objective of the fluorescence microscope has a short $(0.20 \mathrm{~mm})$ working distance. Negative potentials of more than a few kilovolts could therefore result in electric discharges across the sample, destroying it. Extending the bias voltage to the objective lens may succeed in preventing electrical breakdown, but would have the likewise undesirable effect of biasing the fluorescence microscope itself.

In this work, we therefore limit the bias potential to a conservative range as we explore options for better high-voltage shielding and long working distance, high-NA objectives. Figure 1 shows results from a $\sim 0.5$ gigapixel EM acquisition of $80 \mathrm{~nm}$ thin rat pancreas tissue at $3 \mathrm{keV}$ primary electron beam energy 
with $-1.5 \mathrm{kV}$ bias potential and 5us/px dwell. Achieving comparable image quality with the same imaging conditions - excluding the negative bias potential - requires significantly higher electron beam exposure per pixel. Use of the bias potential therefore shows great promise for enhancing throughput in our integrated array tomography procedure, rendering it a viable method for correlative volume EM.

\section{References:}

[1] K Briggman and D Bock, Current Opinion in Neurobiology 22 (2012), p. 154-161.

[2] J Delpiano et al., Journal of Microscopy 271 (2018), p. 109-119.

[3] J Kuipers et al., Experimental Cell Research 337 (2015), p. 202-207.

[4] J Bouwer et al., Advanced Structural Chemical Imaging 2 (2016), p. 11.

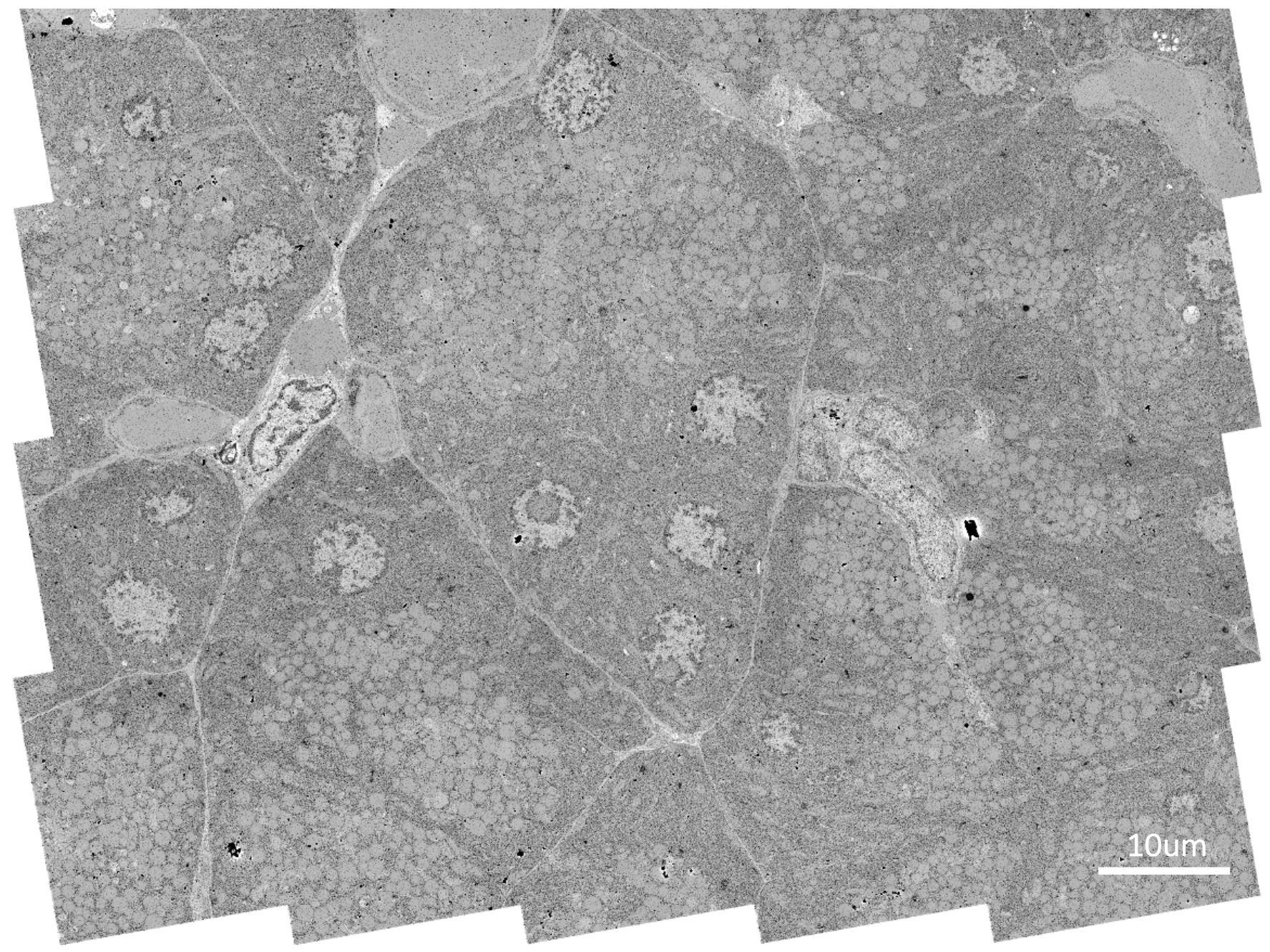

Figure 1. Aligned image following tiled EM acquisition of $80 \mathrm{~nm}$ rat pancreas tissue at $3 \mathrm{keV}$ primary electron beam energy with $-1.5 \mathrm{kV}$ bias voltage applied to the sample. 